\title{
Towards precise relativistic $b$ quarks on the lattice
}

\section{McNeile}

University of Glasgow, Glasgow, UK

E-mail: c.mcneile@physics.gla.ac.uk

\section{C.T.H. Davies}

University of Glasgow, Glasgow, $U K$

E-mail: c.davies@physics.gla.ac.uk

\section{E. Follana*}

Universidad de Zaragoza, Zaragoza, España

E-mail: efollana@unizar.es

\section{K. Hornbostel}

Southern Methodist University, Dallas, TX, USA

E-mail: kjhephysics.smu.edu

\section{G.P. Lepage}

Cornell University, Ithaca, NY, USA

E-mail: g.p.lepage@cornell.edu

\section{J. Shigemitsu}

The Ohio State University, Columbus, Ohio, USA

E-mail: shige@pacific.mps.ohio-state.edu

\section{HPQCD collaboration ${ }^{\dagger}$}

We discuss the status of our ongoing efforts to improve on our calculation of the $D_{s}$ decay constant. We show preliminary results on the ratio of the charm to the strange quark mass. We also present preliminary results for spectroscopy, decay constants and bottom quark mass obtained by performing calculations with highly improved staggered quarks at masses above the c mass and close to the $b$ mass.

The XXVII International Symposium on Lattice Field Theory

July 26-31, 2009

Peking University, Beijing, China

\footnotetext{
*Speaker.

${ }^{\dagger}$ http://www.physics.gla.ac.uk/HPQCD
} 


\section{Introduction}

In the last few years Lattice QCD has become a precision tool, useful for the calculation of non-perturbative, gold-plated quantities in hadronic physics. This allows us to make stringent tests of QCD, and is crucial if we are to use Lattice QCD calculations as an ingredient in the search for physics beyond the Standard Model. It also makes it possible to determine accurately, from first principles, many parameters of the Standard Model (strong coupling constant, quark masses, elements of the CKM matrix.)

Recently it has become possible to include charm quarks accurately in Lattice QCD calculations, thanks to the introduction of the Highly Improved Staggered Quark (HISQ) action [1]. This is a fully relativistic discretization, and has a number of advantages, which we discuss later on, as well as some potential difficulties due to the much higher mass of $\mathrm{c}$ quarks as compared with $\mathrm{u}, \mathrm{d}$ and s quarks. Our results for charm-light decay constants [2], as well as for the charm quark mass [3] show that this approach can produce results with a similar accuracy to the previous staggered light quark calculations [4].

Having seen that it's possible to use this approach with charm quarks, the question immediately comes to mind of whether the same is true for bottom quarks, with ensembles already existing or that can be realistically generated in the near future. In this exploratory study, we increase the mass of the HISQ quarks beyond the charm mass into the bottom mass region.

We briefly discuss general issues with relativistic discretizations of heavy quarks in section 2. We then present in section 3 an update on our calculation of charm-light decay constants, and section 4 presents the status of a calculation of the ratio $m_{c} / m_{s}$, which when combined with our previous accurate value for $m_{c}$ will result in an improved $m_{s}$ determination. In section 5 we discuss preliminary results for masses and decay constants of bottom-bottom and bottom-strange systems, obtained through the relativistic discretization at masses beyond the charm quark mass and an extrapolation in the quark mass to get to the $b$. The same strategy is applied to the calculation of the b quark mass in section 6 . Section 7 presents our conclusions and outlook for future work.

\section{Relativistic Discretization of Heavy Quarks}

The use of the ASQTAD improved staggered discretization in Lattice QCD has made it possible, in recent years, to fully include the effect of $u, d$ and s quarks on the QCD vacuum, resulting in realistic and accurate calculations of many gold-plated properties of hadrons [4]. The ASQTAD discretization removes all tree-level $a^{2}$ errors present in the naive staggered action. Recently we introduced a new, further improved staggered action called HISQ (highly improved staggered quarks). The HISQ action goes beyond $a^{2}$ tree-level improvement by further smearing the gauge fields which enter in the staggered Dirac operator. We showed in [1] that this effectively cuts down the size of the 1-loop taste-changing interactions, which were typically larger than expected in the staggered formulation, as well as the ones liable to cause theoretical concerns if not under control [5].

A relativistic discretization has a number of advantages with respect to an effective heavyquark theory such as NRQCD. If the discretized action has some symmetries, there are quantities which are free from renormalization. For example, for staggered quarks, pseudoscalar meson de- 
cay constants do not renormalize because of PCAC. This eliminates one of the main sources of systematic errors from the calculation. Using the same formulation for the heavy and the light quarks is simpler, and allows us, for example, to calculate accurate ratios of quark masses.

But using a relativistic action for heavy quarks brings forth new issues. Discretization errors will be generally proportional to powers of the quark mass, $(\mathrm{am})^{n}$, and the question is whether those can be kept under control for large masses. For staggered actions only even powers appear, and for an action like HISQ that removes all $a^{2}$ tree-level errors, the discretization errors start at $(\mathrm{am})^{4}$, $\alpha_{s}(a m)^{2}$. These would still be too large for precision work; however, the fact that a system with charm (or heavier) quarks is essentially non-relativistic makes it possible, by a simple modification of the coefficient of the Naik term, to completely remove those errors at leading order in $v / c$, where $v$ is the velocity of the quark inside the hadron [1]. Another essential ingredient is the existence of fine enough ASQTAD sea quark ensembles [6], going from $a \approx 0.15 \mathrm{fm}$ (very coarse), $0.125 \mathrm{fm}$ (coarse), $0.09 \mathrm{fm}$ (fine), $0.06 \mathrm{fm}$ (superfine) and down to $0.045 \mathrm{fm}$ (ultrafine). All these ingredients make it possible to use the HISQ action for calculations with charm quarks with almost the same level of precision as was possible for light quarks. The existence of ensembles at several lattice spacings is crucial to reliably extrapolate to the continuum limit. For bottom quarks, at present we must also extrapolate in the heavy quark mass: we do calculations at several values of the lattice spacing and the heavy quark mass (above the charm mass), and make a joint $a \rightarrow 0, M_{h} \rightarrow M_{b}$ extrapolation. On the ultrafine ensemble such extrapolation is small.

\section{3. $f_{D_{s}}$ decay constant}

Our prediction of the $D$ and $D_{s}$ decay constants caused a lot of interest because experimental determinations soon after $[7,8,9]$ gave a surprising picture: $f_{D}$ agreed very well with our result but $f_{D_{s}}$ was more than $3 \sigma$ higher $[10,11]$. Here $\sigma$ was dominated by the experimental error since our own error was so small. Because we use a relativistic discretization in which there is a PCAC relation, we can calculate $f_{D}$ and $f_{D_{s}}$ from the partially conserved axial current, in exactly the same way as is done for $f_{\pi}$ and $f_{K}$ (for which we agree with the experimental values). Using three values of the lattice spacing, we obtained a $2 \%$ accurate result. We now have extended the calculation to two finer lattice spacings, the superfine $a \approx 0.06 \mathrm{fm}$, and the ultrafine $a \approx 0.045 \mathrm{fm}$. We show in Fig. 1 the preliminary results from this updated calculation, and a comparison of our data with current experimental determinations which have changed the picture considerably from that of last year. Now new CLEO determinations $[12,13]$ are closer to our result for $f_{D_{S}}$ (especially for some channels) and a reanalysis of $\mathrm{BaBar}$ data [14] is very close indeed. The discrepancy between our calculation and the current world average given by the Heavy Flavor Averaging Group is only about $2 \sigma[15] .{ }^{1}$.

\section{Ratio of $m_{c}$ to $m_{s}$}

Because we are using the same HISQ action for all quarks, light and heavy, we can obtain very

\footnotetext{
${ }^{1}$ In fact the final discrepancy should be even a little smaller, because our final result will use the updated determination of the parameter $\mathrm{r}$ [16], which is used to set the scale and therefore the physical value of the lattice spacing. r1 has decreased a little from its previous calculated value, and that in turn will increase slightly the value of $f_{D_{s}}$.
} 

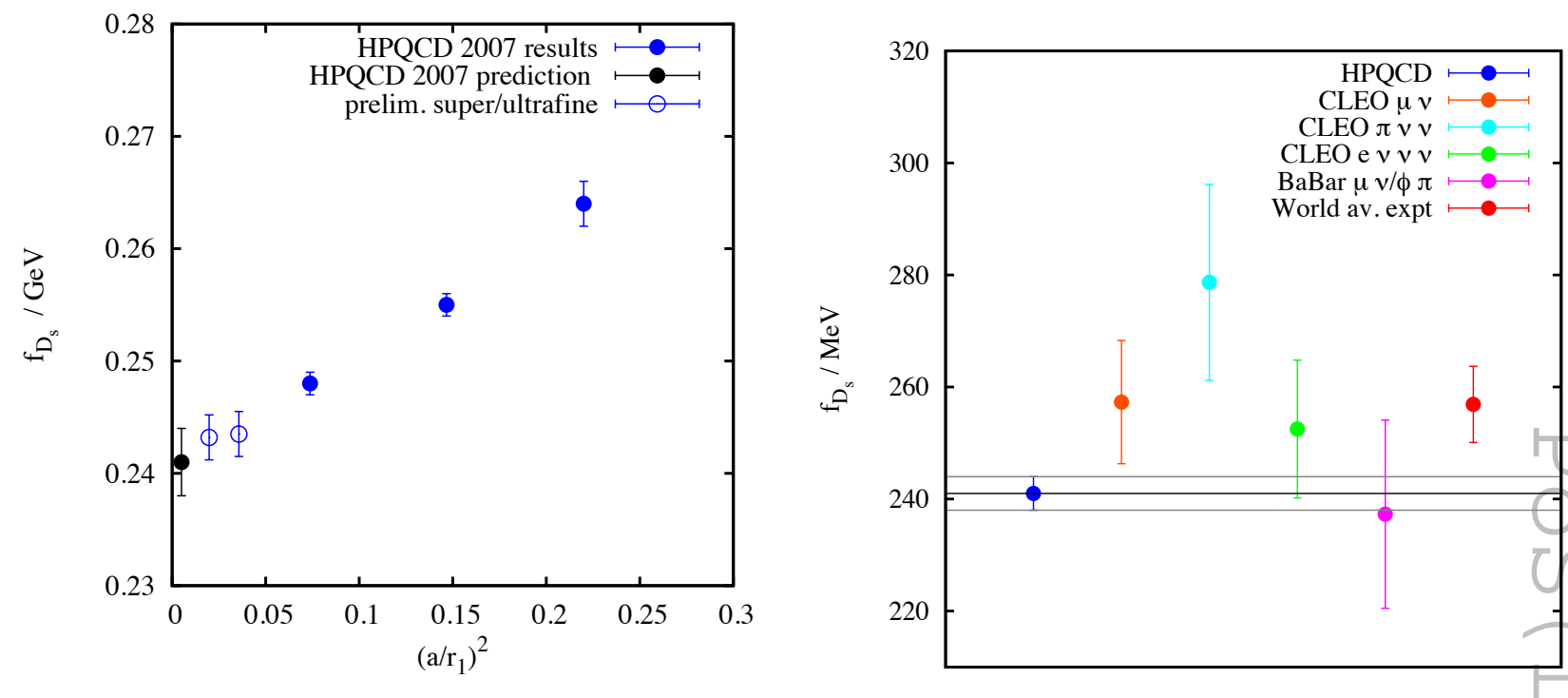

Figure 1: HPQCD lattice calculation and comparison with experiment. World average is from HFAG [15]

accurate ratios of quark masses, where some systematic errors cancel. In particular, we have a very precise value for the ratio of lattice masses, $m_{c} / m_{s}$. We can now go to another scheme, like $\overline{M S}$, and the $\mathrm{Z}$ factors that appear will cancel in the ratio; this allows us to combine this ratio with our precision calculation of the $m_{c}$ mass [3] to obtain a determination of the strange quark mass with a $\approx 1.5 \%$ total error.

In Fig. 2 we show preliminary results for $m_{c} / m_{s}$. The gray points in the plot are the lattice results for the ratio at various bare masses; the blue points are the results interpolated to the physical masses, determined by fixing the masses of the $\eta_{c}$ and the $\eta_{s}$ to their physical values ${ }^{2}$; the red point is the continuum and chirally extrapolated value.

\section{Masses and decay constants for bottom-bottom and bottom-strange systems}

In order to study bottom quarks with current lattices, we must resort to an extrapolation procedure: we calculate at several values of the lattice spacing $a$ and masses above the charm mass, $M_{h}$, and then we do a joint continuum and mass extrapolation, $a \rightarrow 0, M_{h} \rightarrow M_{b}$.

In Fig. 3 we show preliminary results for the difference between the masses of the heavystrange and the heavy-heavy pseudoscalar mesons, $\Delta M_{h s}=M_{h s}-M_{h h} / 2$, for a range of masses from the charm to the bottom quark mass and for several lattice spacings. We can see that the results have a smooth dependence on both the heavy mass and the lattice spacing, and the extrapolated value to the bottom mass will be consistent with experiment.

In Fig. 4 we show similar data for the decay constant of the heavy-strange pseudoscalar, $f_{h s}$, which interpolates between $f_{D_{s}}$ and $f_{B s}$. The extrapolated value should be consistent, and already

\footnotetext{
${ }^{2}$ See [16] for a discussion on what we mean by the "physical" value of $\eta_{s}$; the main point is that it can be used to accurately fix the strange quark mass.
} 


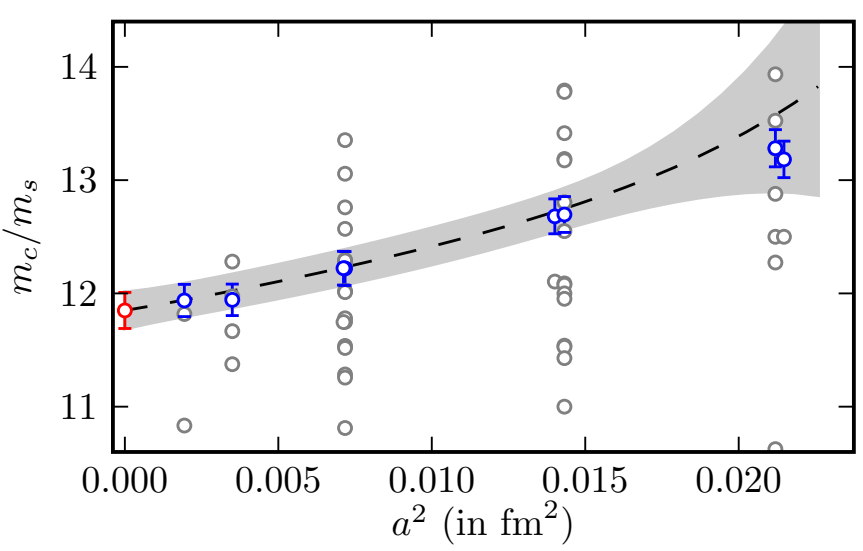

Figure 2: Ratio of charm to strange quark masses.

competitive, with the previous NRQCD calculation [17].

\section{Bottom quark mass}

Another thing which we can do with the relativistic formulation is a calculation of the bottom quark mass, with the same methods used for the charm quark mass calculation [3]. Let's recall that the basic idea is to take advantage of the high-order continuum QCD perturbation calculations performed by Chetyrkin, Kühn, Steinhauser and Sturm, by combining them with high precision lattice data for moments of pseudoscalar and vector current correlators. For the case of a pseudoscalar current, the basic object is the correlator:

$$
G(t) \equiv a^{6} \sum_{\mathbf{x}}\left(a m_{0 h}\right)^{2}\left\langle 0\left|j_{5}(\mathbf{x}, t) j_{5}(0,0)\right| 0\right\rangle, j_{5}=\bar{\psi}_{h} \gamma_{5} \psi_{h}
$$

which is finite and unrenormalized as $a \rightarrow 0$, due to PCAC. The quantities to compare with continuum PT are the moments, $G_{n}=\sum_{t}(t / a)^{n} G(t)$, of such correlator, for which we have:

$$
G_{n}(a=0)=\frac{g_{n}\left(\alpha_{\overline{M S}}(\mu), \mu / M_{h}\right)}{\left(a M_{h}(\mu)\right)^{n-4}}
$$

Here the $g_{n}$ are known to large orders in continuum PT for large $M_{h}$ and not too large $n$, and the left hand side is calculated from the lattice data, giving us the value of $a M_{h}$. In practice we use what are called reduced moments, in order to remove some of the discretization errors (see [3] for details.)

In Fig. 5 we can see several reduced moments, for different values of the heavy-heavy pseudoscalar mass and several values of the lattice spacing, as well as the continuum extrapolations. With this method we expect the final value on $m_{b}$ to have a total error of $2-3 \%$.

\section{Conclusions and Outlook}

It is feasible to use a highly improved relativistic action for charm quarks on fine enough lattices. This allows us to do very accurate calculations for systems with charm quarks. We obtain 


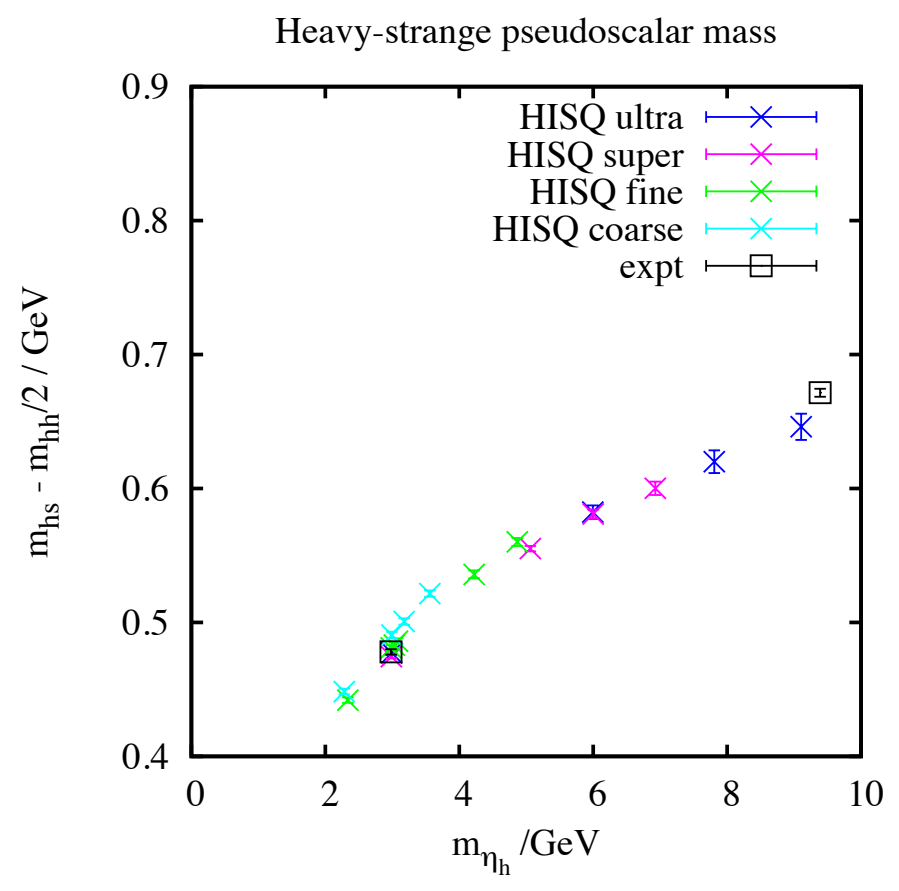

Figure 3: $\Delta M_{h s}=M_{h s}-M_{h h} / 2$

a $2 \%$ accurate result for $f_{D_{s}}$. Due to changes in the experimental values, our calculation is now only about $2 \sigma$ away from experiment. We also can obtain a very accurate $m_{c} / m_{s}$ mass ratio, which combined with our $1 \% m_{c}$ determination will provide a $\approx 1.5 \%$ determination of $m_{s}$.

To get to the bottom quark mass, we need (at least for now) to extrapolate in the mass of the heavy quark. This can be done, as we show for $\Delta M_{b s}=M_{b s}-M_{b b} / 2$ and $f_{B_{s}}$, and it seems that the additional source of error that it introduces can be kept under control. The results are in both cases consistent with either experiment or previous NRQCD calculations.

If we had even finer lattices $(0.03 \mathrm{fm}$, say) we could reduce substantially that uncertainty. This may be a good strategy to reduce the errors on some B physics quantities, for example $f_{B_{s}}$.

\section{Acknowledgments}

We are grateful to the MILC collaboration for the use of their configurations. Computing was done at USQCD's Fermilab cluster, the Ohio Supercomputer Center and the Argonne Leadership Computing Facility at Argonne National Laboratory, which is supported by the Office of Science of the U.S. Department of Energy under contract DOE-AC02- 06CH11357. We acknowledge the use of Chroma [18] for part of our analysis. This work was supported by the Leverhulme Trust, 


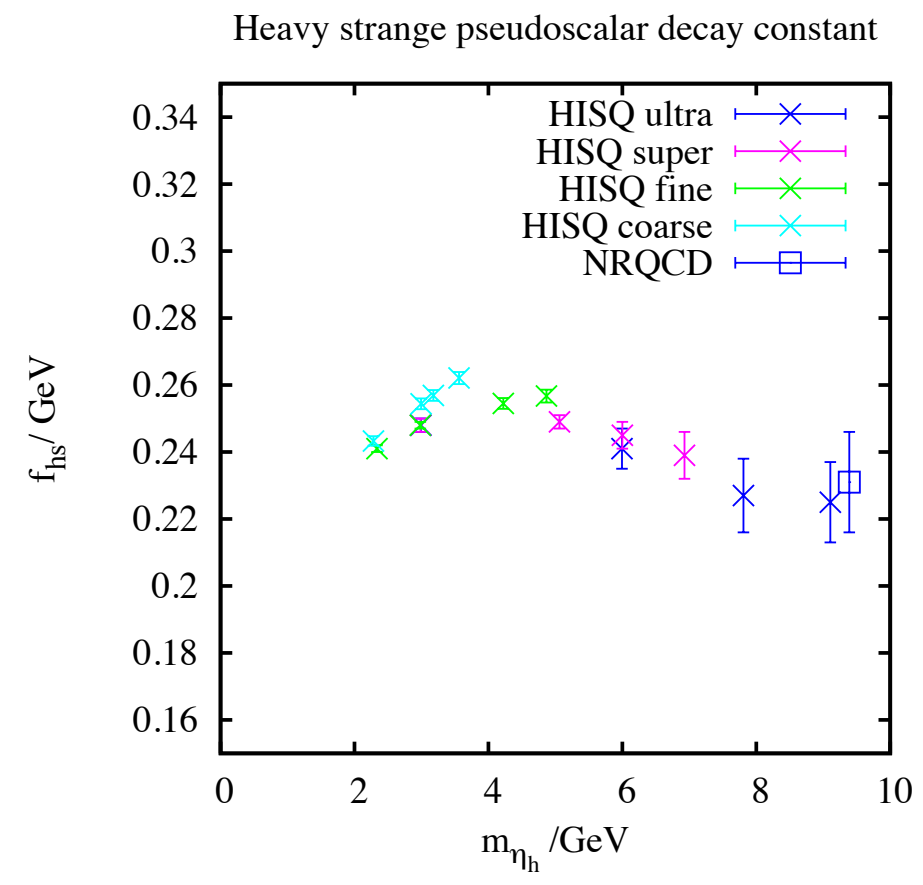

Figure 4: $f_{h s}$

NSF, the Royal Society, the Scottish Universities Physics Alliance, STFC and DOE. E. Follana is supported by Ministerio de Ciencia e Innovación through the Ramón y Cajal program.

\section{References}

[1] E. Follana et al, HPQCD collaboration, Phys. Rev. D 75:054502 (2007) [hep-lat / 0610092 ].

[2] E. Follana, C.T.H. Davies, G.P. Lepage and J. Shigemitsu, HPQCD collaboration, Phys. Rev. Lett. 100:062002 (2008) [arXiv:0 706.1726 [hep-lat] ].

[3] I. Allison et al, HPQCD collaboration + K.G. Chetyrkin, J.H. Kühn, M. Steinhauser and C. Sturm, Phys.Rev.D 78:054513 (2008) [arXiv: 0805.2999 [hep-lat]].

[4] C.T.H. Davies et al, Fermilab Lattice/HPQCD/MILC collaborations, Phys. Rev. Lett. 92:022001 (2004) [hep-lat/0304004].

[5] S.R. Sharpe, PoSLAT2006:022 (2006) [arXiv: hep-lat/0610094].

[6] C. Bernard et al, Phys.Rev. D 64:054506 (2001) [hep-lat/ 0104002 ].

[7] K.M. Ecklund, et al, CLEO-c, Phys.Rev.Lett.100:161801, (2008) [arXiv:0 712 .1175].

[8] B.I. Eisenstein et al, CLEO-c, Phys.Rev.D78:052003, (2008) [arXiv: 0806.2112]. 


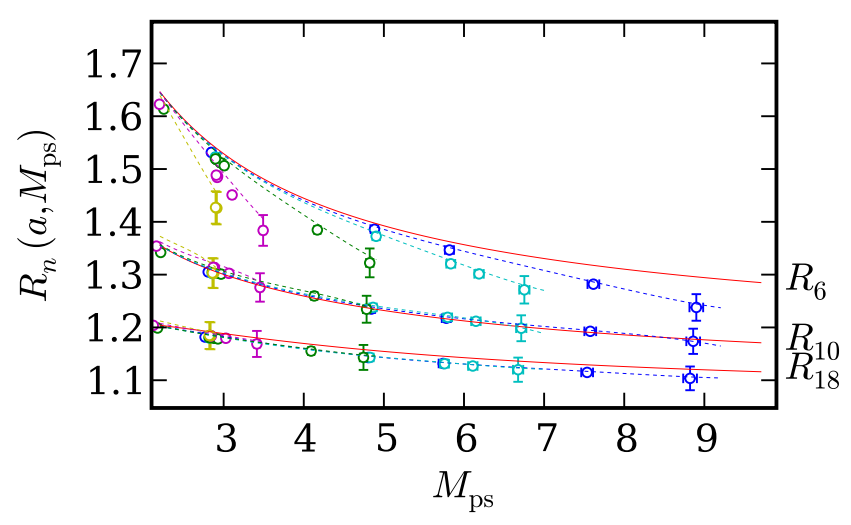

Figure 5: 6th, 10th and 18th reduced moments of the pseudoscalar current-current correlator.

[9] Liming Zhang, CLEO-c, [arXiv: 0810.2328].

[10] E. Gamiz, arXiv:0811.4146.

[11] C.T.H. Davies et al, HPQCD collaboration, arXiv:0810.3548.

[12] P.U.E. Onyisi, et al, CLEO-c, Phys.Rev.D79:052002, (2009) [arXiv: 0901.1147 ].

[13] J.P. Alexander, et al, CLEO-c, Phys.Rev.D79:052001, (2009) [arXiv: 0901.1216 ].

[14] A.J. Schwartz, arXiv:0909.4473.

[15] http://www.slac.stanford.edu/xorg/hfag/index.html

[16] C.T.H. Davies, E. Follana, I.D. Kendall, G. Peter Lepage, C. McNeile, HPQCD collaboration, arXiv:0910.1229.

[17] E. Gámiz, C.T.H. Davies, G.P. Lepage, J. Shigemitsu, M. Wingate, HPQCD collaboration, Phys.Rev.D 80:014503, (2009) [arXiv: 0902 .1815].

[18] R.G. Edwards and B. Joo [SciDAC/LHPC/UKQCD Collaborations], Nucl. Phys. Proc. Suppl. 140832 (2005) [arXiv: hep-lat/0409003]. 